\title{
Clinical Predictors of Oro-esophageal Tube Feeding Success in Brain Injury Patients With Dysphagia
}

\author{
Yoon Mok Chun, $\mathrm{MD}^{1}$, Min Ho Chun, MD, PhD ${ }^{1}$, Kyung Hee Do, MD, PhD ${ }^{2}$, Su Jin Choi, MD $^{1}$ \\ ${ }^{1}$ Department of Rehabilitation Medicine, Asan Medical Center, University of Ulsan College of Medicine, Seoul; \\ ${ }^{2}$ Department of Physical Medicine and Rehabilitation, Veterans Health Service Medical Center, Seoul, Korea
}

\begin{abstract}
Objective To identify possible clinical predictors of intermittent oro-esophageal (OE) tube feeding success, and evaluate the clinical factors associated with $\mathrm{OE}$ tube treatment.

Methods A total of 135 dysphagic patients were reviewed, who received OE tube treatment and were hospitalized in the department of rehabilitation medicine between January 2005 and December 2014. The 76 eligible cases enrolled were divided into two groups, based on the OE tube training success. Clinical factors assessed included age, cause of brain lesion, gag reflex, cognitive function and reasons for $\mathrm{OE}$ tube training failure.

Results Of the 76 cases enrolled, 56 study patients were assigned to the success group, with the remaining 20 in the failure group. There were significant differences between these two groups in terms of age, gag reflex, ability to follow commands, and the score of Korean version of Mini-Mental Status Examination (K-MMSE). Location of the brain lesion showed a borderline significance. Multivariable analysis using logistic regression revealed that age, cause of brain lesion, gag reflex, and K-MMSE were the main predictors of OE tube training success.

Conclusion A younger age, impaired gag reflex and higher cognitive function (specifically a K-MMSE score 219.5 ) are associated with an increased probability of $\mathrm{OE}$ tube training success in dysphagic patients.
\end{abstract}

Keywords Brain injuries, Dysphagia, Oro-esophageal tube, Clinical predictor, Multivariate analysis

\section{INTRODUCTION}

Dysphagia is a common condition after brain injury. Previous reports using videofluoroscopic swallowing study (VFSS) estimated the dysphagia prevalence to be
$35 \%$ to $67 \%$ in stroke patients. It is also estimated that $30 \%$ of traumatic brain injury (TBI) patients have a swallowing disorder [1-3]. Mukand et al. [4] found that $26 \%$ of their brain tumor patients had dysphagia. Dysphagia may cause aspiration pneumonia, under-nutrition, and/or a

Received November 30, 2016; Accepted February 3, 2017

Corresponding author: Min Ho Chun

Department of Rehabilitation Medicine, Asan Medical Center, University of Ulsan College of Medicine, 88 Olympic-ro 43-gil, Songpa-gu, Seoul 05505, Korea. Tel: +82-2-3010-3800, Fax: +82-2-3010-6964, E-mail: mhchun@amc.seoul.kr

ORCID: Yoon Mok Chun (http://orcid.org/0000-0002-8274-215X); Min Ho Chun (http://orcid.org/0000-0001-8666-7225); Kyung Hee Do (http://orcid. org/0000-0003-4235-8759); Su Jin Choi (http://orcid.org/0000-0002-3443-2237).

(c) This is an open-access article distributed under the terms of the Creative Commons Attribution Non-Commercial License (http://creativecommons.org/ licenses/by-nc/4.0) which permits unrestricted noncommercial use, distribution, and reproduction in any medium, provided the original work is properly cited. Copyright (c) 2017 by Korean Academy of Rehabilitation Medicine 
decreased quality of life [5]. Since there is an increase in the morbidity and mortality because of complications arising due to dysphagia, a proper feeding method is required in the affected patients. Approximately 13\%-15\% of stroke patients show a persistent swallowing dysfunction after 6 months [6], and many cases of severe dysphagia remain dependent on tube feeding for longer periods [7].

The common methods of enteral tube feeding include nasogastric (NG) and gastrostomy tubes. NG tube feeding is the most common; the tube is easy to insert and has been used successfully for enteral nutrition. However, this feeding is associated with complications such as rhinitis, esophageal reflux, strictures, aspiration pneumonia, and esophagitis, and the risk of tube misplacement also indicates that NG tube is not an ideal long-term method of tube feeding [8]. The gastrostomy tube is ideally offered to patients requiring long term (longer than 4 weeks) tube feeding, but associated complications such as peristomal infection, peritonitis, inadvertent removal, and leakage [9].

Intermittent oro-esophageal (OE) tube feeding was introduced in 1988 by Campbell-Taylor et al. [10], to supply nutrition to patients with dysphagia. In the OE tube method, the feeding tube is intermittently inserted into the esophagus. Previous studies have reported that the OE tube improves swallowing functions and reduces the risk of pneumonia, reflux and aspiration [10-12]. In addition, studies indicate the $\mathrm{OE}$ tube to be a safer tube feeding method that can be applied over a longer period without severe complications $[10,13,14]$. However, since there are no clear optimal guidelines for the indication of OE tubes in dysphagic patients with a brain lesion, there are difficulties in selecting appropriate dysphagic brain lesion patients for OE tube treatment. The aim of this study was to evaluate the clinical factors associated with OE tube training success and identify possible clinical predictors.

\section{MATERIALS AND METHODS}

\section{Subjects}

Dysphagic patients hospitalized in the department of rehabilitation medicine and who had undergone OE tube training in the period between January 2005 and December 2014 were retrospectively identified. A total of 135 patients underwent swallowing therapy at our hospital using an OE tube. Patients with a brain lesion (stroke, brain tumor, and TBI) were included in our current analysis. We excluded patients who met any of the following criteria: (1) active medical problem such as respiratory infection, (2) no VFSS evaluation during admission or no VFSS-confirmed dysphagia, (3) verified abnormal findings in the esophageal phase of VFSS, (4) history of head and neck cancer or operation, or (5) inability to appropriately respond to verbal commands. From an initial cohort of 135 patients, 76 patients were finally recruited. All patients included in this study were assigned to either an $\mathrm{OE}$ tube success or failure group. The success group included patients where an OE tube was successfully and safely inserted at least 3 times, or who were capable of attempting $\mathrm{OE}$ tube feeding. The failure group contained patients in whom the $\mathrm{OE}$ tube could not be inserted for various reasons.

Data was collected from the medical records of our study cases, including age, sex, onset (duration between onset and $\mathrm{OE}$ tube training), cause of brain lesion, location of brain lesion, and history of pneumonia. We assessed the ability of these patients to follow commands, their Korean version of Mini-Mental Status Examination (K-MMSE) score for cognitive function, VFSS findings, reason for OE tube training failure, and any OE tube complications. This present study was approved by Asan Medical Center Institutional Review Board and local ethics committee (IRB No. S2014-2101-0002).

\section{OE tube protocol}

OE tubes were applied to patients who met the following criteria: (1) dysphagia with non-oral feeding or severely poor oral intake, (2) an alert consciousness, (3) no medical problems such as pneumonia, and (4) ability to adhere to OE tube procedure. An 8-12 French Nelaton tube (effective tube length $360 \mathrm{~mm}$, natural rubber latex catheter) was used for the OE tube treatment and feeding procedure. In the first $\mathrm{OE}$ tube treatment session, an occupational therapist inserted the tube. The Nelaton tip was first immersed in water to reduce friction. Insertion proceeded with the patient's chin facing down to prevent entry into the airway. The patient was asked to swallow the tube when the tube tip reached the lateral wall of the pharynx. The tube was then inserted carefully through the pharynx as far as possible without triggering the gag 
reflex. Tube insertion was stopped if there was a possibility of incorrect insertion, or if any of the following events occurred: (1) voice change, (2) coiling of the tube in the mouth, (3) coughing or signs of choking, or (4) a severe gag reflex.

To ensure correct tube placement, the end of the tube was put in a glass of water after insertion to check for bubbles due to the pressure of the cricopharyngeus or esophageal peristalsis, and siphoning due to the negative pressure of the pharynx. Five respiratory cycles were observed. We then injected $10 \mathrm{~mL}$ of air through the OE tube to check whether the patient coughed, and a further 2-3 mL of water to check for any signs of aspiration. Patients and caregivers were both shown how to insert the OE tube. Once the patient was able to safely insert the tube, OE tube feeding commenced in the ward. All patients continued conventional swallowing therapy.

\section{Data analysis}

Clinical factors, neuroanatomical characteristics, and the gag reflex were compared between the OE tube success and failure groups, using the Mann-Whitney test and Fisher exact test. A multivariable logistic model was applied to identify independent clinical predictors of $\mathrm{OE}$ tube training success (odds ratios and $95 \%$ confidence interval [CI]). A p-value $<0.05$ was considered statistically significant. A receiver operating characteristic (ROC) curve was used to determine the optimal cutoff values of some predictive variables (the area under the curve [AUC] $>0.5$ ). All analyses were performed using SPSS ver. 18.0 (SPSS Inc., Chicago, IL, USA).

\section{RESULTS}

The clinical characteristics of the two study groups are

Table 1. Characteristics of the study patients

\begin{tabular}{|lccc}
\hline \multicolumn{1}{c}{ Variable } & Success group (n=56) & Failure group (n=20) & p-value \\
\hline Sex & & & 0.10 \\
\hline Male & 42 & 11 & \\
\hline Female & 14 & $67.1 \pm 12.6$ & $0.03^{*}$ \\
\hline Age (yr) & $60.1 \pm 10.5$ & $97.9 \pm 114.8$ & 0.76 \\
\hline Duration after onset (day) & $110.0 \pm 229.6$ & & 0.27 \\
\hline Cause & & 13 & \\
\hline Stroke & 46 & 3 & 0.06 \\
\hline Brain tumor & 5 & 4 & \\
\hline Traumatic brain injury & 5 & $10(50.0)$ & 0.43 \\
\hline Location of brain lesion & $15(26.8)$ & $10(50.0)$ & $0.04^{*}$ \\
\hline Supra-tentorial & $41(73.2)$ & $5(25.0)$ & \\
\hline Infra-tentorial & $11(19.6)$ & & \\
\hline History of pneumonia & & $9(45.0)$ & \\
\hline Gag reflex & $9(16.1)$ & $7(35.0)$ & 0.05 \\
\hline Normal & $30(53.6)$ & $4(20.0)$ & \\
\hline Decreased & $17(30.3)$ & $3(15)$ & \\
\hline Absent & $3(5.4)$ & $5(25)$ & 0.05 \\
\hline Follow commands & $7(12.5)$ & $12(60)$ & $18.9 \pm 9.4$ \\
\hline 1 step & $46(82.1)$ & $23.8 \pm 7.6$ & \\
\hline 2 steps & & \\
\hline 3 steps & & & \\
\hline K-MMSE score & & & \\
\hline
\end{tabular}

Values are presented as number (\%) or mean \pm standard deviation.

Onset, duration between onset and oro-esophageal tube training; K-MMSE, Korean version of the Mini-Mental Status Examination.

${ }^{*} \mathrm{p}<0.05$, Fisher exact test, linear by linear association. 
presented in Table 1. There were significant differences between the groups in terms of age, gag reflex, ability to follow commands, and K-MMSE $(\mathrm{p}<0.05)$. The mean age of the success group was $60.1 \pm 10.0$ years, which was 7.0 years younger than that of the failure group $(67.1 \pm 12.6$ years). Gag reflex was classified as normal, decreased, or absent. There were $16 \%$ normal gag reflex patients in the success group, but $45 \%$ in the failure group. There were no significant differences between the unilateral and bilateral gag reflex impaired patients. The mean K-MMSE scores of the success and failure groups were 23.8 \pm 7.6 and 18.9 \pm 9.4 , respectively. The success group patients were able to follow more command steps than patients in the failure group. There were no significant differences in the cause of brain lesion. Infra-tentorial lesions were non-significantly associated with successful OE tube training compared with supra-tentorial lesions, but showed statistical trends of more OE tube success than supra-tentorial lesions.

Multiple logistic regression analysis of the clinical features of the dysphagic patients revealed that age $(p=0.003)$, cause of brain lesion $(p=0.021)$, gag reflex $(\mathrm{p}=0.037)$ and K-MMSE $(\mathrm{p}=0.030)$ were independent predictors of successful $\mathrm{OE}$ tube training (Table 2). A younger age was also associated with $\mathrm{OE}$ tube training success. Patients with a brain tumor or TBI showed a higher failure rate than patients with stroke. Impaired gag reflex and a higher K-MMSE markedly increased the rate of $\mathrm{OE}$ tube insertion success.

Given that age and the K-MMSE cutoff values predicted OE tube success, we assessed the ROC curve (Table 3, Fig. 1). We were unable to determine the cutoff value for age, since the AUC was less than 0.5. The AUC for KMMSE was 0.67, with a standard error of 0.072 (95\% CI, 0.526-0.807; $\mathrm{p}=0.028$ ). The K-MMSE cutoff score associated with successful $\mathrm{OE}$ tube training was 19.5.

The reasons for OE tube failure were gag reflex (42.1\%), refusal or noncooperation (31.6\%), and inability to swallow the tube (26.3\%). Only one patient developed an OE tube associated complication, specifically a sore throat. However, this patient was in the success group and maintained feeding with the OE tube.

Table 2. Multiple logistic regression analysis for independent predictive clinical factors of oro-esophageal tube failure

\begin{tabular}{|c|c|c|c|}
\hline & Number of failure & OR (95\% CI) & p-value \\
\hline Total $(\mathrm{n}=76)$ & $20(26.3)$ & & \\
\hline Age & & $1.12(1.04-1.21)$ & $0.003^{*}$ \\
\hline \multicolumn{4}{|l|}{ Cause } \\
\hline Stroke $(\mathrm{n}=59)$ & $13(22.0)$ & 1.0 & $0.021^{*}$ \\
\hline Brain tumor $(\mathrm{n}=8)$ & $3(37.5)$ & $39.07(2.88-530.03)$ & \\
\hline Traumatic brain injury $(n=9)$ & $4(44.4)$ & $1.74(0.25-12.17)$ & \\
\hline \multicolumn{4}{|l|}{ Gag reflex } \\
\hline Normal gag reflex $(\mathrm{n}=18)$ & $9(45.0)$ & 1.0 & $0.037^{*}$ \\
\hline Decreased gag reflex $(n=37)$ & $7(35.0)$ & $0.15(0.03-0.72)$ & \\
\hline Absent gag reflex $(n=21)$ & $4(20.0)$ & $0.16(0.03-0.87)$ & \\
\hline K-MMSE score & & $0.92(0.85-0.99)$ & $0.030^{*}$ \\
\hline
\end{tabular}

Values are presented as number (\%) or mean \pm standard deviation.

OR, odds ratio; CI, confidence interval; K-MMSE, Korean version of the Mini-Mental Status Examination.

${ }^{*} \mathrm{p}<0.05$, correlations among multiple variables and oro-esophageal tube failure by multiple logistic regression analysis.

Table 3. Cutoff values for age and K-MMSE using ROC curve analysis

\begin{tabular}{lcccc}
\hline & Area under the curve & Cutoff value & Sensitivity & Specificity \\
\hline K-MMSE score & 0.67 & 19.5 & 0.80 & 0.50 \\
Age & 0.32 & - & - & - \\
\hline
\end{tabular}

K-MMSE, Korean version of the Mini-Mental Status Examination; ROC, receiver operating characteristic. 


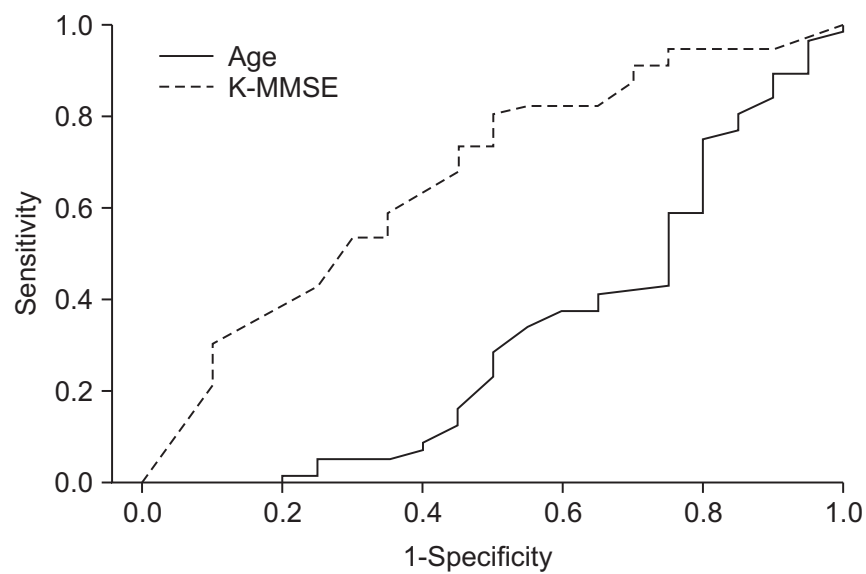

Fig. 1. The area under the ROC curve (AUC) analysis for the score of Korean version of the Mini-Mental Status Examination (K-MMSE) and age.

\section{DISCUSSION}

This study investigated the clinical factors predicting OE tube training success in dysphagic patients with a brain lesion. We found that age, stroke (cause of brain lesion), impaired gag reflex, and higher cognitive function are predictive of $\mathrm{OE}$ tube training success.

In our current study, the cause of brain injury was classified into stroke, brain tumor, and TBI. TBI patients had a higher rate of OE tube failure. In addition, since TBI patients showed the most impaired cognitive functions and lowest abnormal gag reflex percentage, the results of these patients were as expected. The $\mathrm{OE}$ tube failure rate of tumor patients was also very high. However, because the brain tumor patients were younger, had a near normal cognitive function and a high rate of abnormal gag reflex, this outcome was contrary to our expectations. Notably, there were only 3 tumor patients in the failure group, which probably limited the analysis. Thus, a larger sample size is required in future, to identify the OE tube success rate of tumor patients.

The gag reflex is a normal defense mechanism that prevents foreign bodies from entering the upper respiratory tract [15]. Because the OE tube is intermittently inserted through the oral cavity into the esophagus, this reflex can lead to OE tube feeding failure. In our present study, the most common reason for $\mathrm{OE}$ tube failure was indeed found to be the gag reflex. A greater number of our patients had a decreased or absent gag reflex in the success group than in the failure group (Fig. 2). Both, a bilaterally

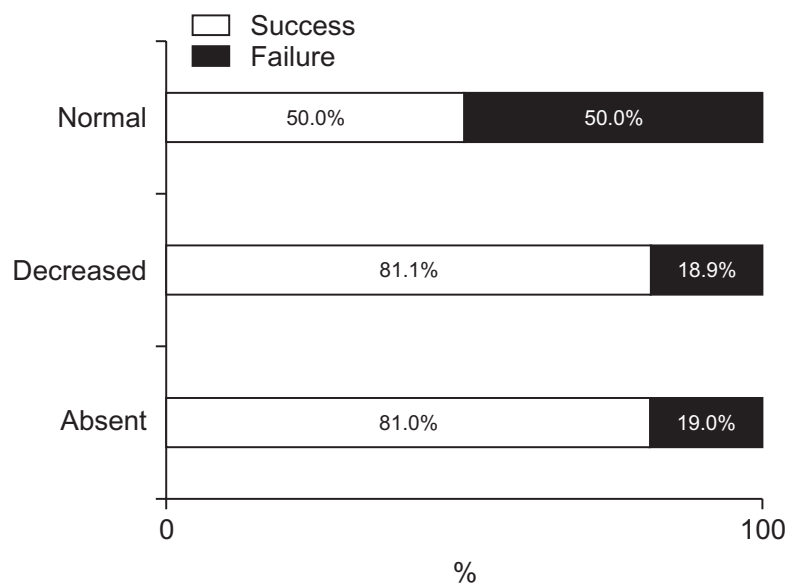

Fig. 2. Success rate, according to gag reflex characteristics.

and unilaterally impaired gag reflex, are associated with a higher $\mathrm{OE}$ tube success rate compared with a normal gag reflex in our current series. This finding indicates that a unilaterally impaired gag reflex mechanism also satisfactorily enables the patient to withstand the intraoral stimuli caused by the OE tube. Although a gag reflex is often absent in healthy adults [16], an absent gag reflex is 10 times more frequent in dysphagic patients with an acute stroke, and is associated with a need for tube feeding [17]. It is reported to be an independent predictor of non-oral feeding in the subacute phase (at 6 weeks from onset) [18]. Brain lesion patients with an impaired gag reflex have a higher chance of requiring long term tube feeding. However, although the gastrostomy tube, and not the NG tube, is the ideal method for patients requiring longterm enteral feeding, the complications and invasiveness of the stomal site leads to resistance in some patients and their care givers. Moreover, a continuously inserted NG or gastrostomy tube constrains patients from returning to their daily routines and work. Thus, it is worthwhile to utilize OE tube feeding for dysphagic patients with an abnormal gag reflex.

Previous studies have reported that patients treated with the OE tube were required to understand this procedure, be cooperative, and show proper cognitive function $[10,12-14,19]$. In our present study series, a higher cognitive function was associated with higher OE tube training success. The reason for this association is that the training requires the perception, attention, memory, and executive functions of the patient, and also because a severely decreased cognitive function correlates with 
chewing and swallowing dysfunction $[20,21]$. However, no study to date has recommended that any specific level of cognitive function is required for successful $\mathrm{OE}$ tube training. Here, we have used the ROC curve analysis to determine an optimal K-MMSE cutoff score of 19.5.

There was no significant association with the location of brain lesion and tube feeding success in our current cohort. This is possibly due to a biased selection of supra-tentorial patients who would be more likely to successfully complete OE tube training than other supratentorial patients. The enrolled supra-tentorial patients in our current study were more likely to have a greater gag reflex impairment or higher cognitive function, which are better conditions for OE tube training. Another possible reason was an uneven proportion of infra-tentorial patients in our study population. This bias seems to have occurred due to the widespread belief that infra-tentorial patients might be more suitable for OE tube training. Indeed, our infra-tentorial group showed higher cognitive functions than the supra-tentorial group (K-MMSE: $25.6 \pm 5.9$ vs. $16.0 \pm 9.0$ ), and a higher abnormal gag reflex rate (abnormal gag reflex: $81.4 \%$ vs. $68.0 \%$ ). These findings are in line with statistical trends for greater OE tube success in the infra-tentorial group ( $\mathrm{OE}$ tube success rate: $80.4 \%$ vs. $60.0 \%$ ). Based on these findings, we believe that statistically meaningful results would be obtained with a larger number of patients in a future investigation.

Since ours was a retrospective study, it had several noteworthy limitations. The first was that the K-MMSE scores were not assessed at the starting point of OE tube training. The K-MMSE was performed at admission or transfer, which was before the time the OE tube training starting point. Thus, the score obtained might not exactly reflect the cognitive function of patients at the OE tube training point. However, 20 of our study patients were in the chronic phase and stationary state. In the other 56 patients, the mean interval between the K-MMSE and the OE tube training start time was less than 1 week $(6.75 \pm 5.55$ days). Therefore, it is likely that there was no significant difference in cognitive function between these 2 points. Second, although the gag reflex was routinely checked during OE tube treatment, it was not examined by a predetermined physician or occupational therapist. Third, the OE tube training process administered to each patient was not uniformly controlled.

Notwithstanding these aforementioned limitations, our current study is, to our knowledge, the first report to compare the clinical factors associated with $\mathrm{OE}$ tube training success, and to identify the clinical predictors of OE tube feeding success. A well-controlled prospective study is needed to confirm our findings.

In conclusion, the OE tube feeding method can be applied to brain lesion patients with dysphagia. Successful OE tube training is associated with a younger age, an impaired gag reflex and higher cognitive function (particularly, a K-MMSE score 219.5 ). These factors should therefore be considered in order to increase the probability of $\mathrm{OE}$ tube training success when considering OE tube feeding of dysphagic patients.

\section{CONFLICT OF INTEREST}

No potential conflict of interest relevant to this article was reported.

\section{REFERENCES}

1. Field LH, Weiss CJ. Dysphagia with head injury. Brain Inj 1989;3:19-26.

2. Takizawa C, Gemmell E, Kenworthy J, Speyer R. A systematic review of the prevalence of oropharyngeal dysphagia in stroke, Parkinson's disease, Alzheimer's disease, head injury, and pneumonia. Dysphagia 2016;31:434-41.

3. Wesling M, Brady S, Jensen M, Nickell M, Statkus D, Escobar N. Dysphagia outcomes in patients with brain tumors undergoing inpatient rehabilitation. Dysphagia 2003;18:203-10.

4. Mukand JA, Blackinton DD, Crincoli MG, Lee JJ, Santos BB. Incidence of neurologic deficits and rehabilitation of patients with brain tumors. Am J Phys Med Rehabil 2001;80:346-50.

5. Nishiwaki K, Tsuji T, Liu M, Hase K, Tanaka N, Fujiwara $\mathrm{T}$. Identification of a simple screening tool for dysphagia in patients with stroke using factor analysis of multiple dysphagia variables. J Rehabil Med 2005; 37:247-51.

6. Mann G, Hankey GJ, Cameron D. Swallowing function after stroke: prognosis and prognostic factors at 6 months. Stroke 1999;30:744-8.

7. Kirby DF, Delegge MH, Fleming CR. American Gastroenterological Association technical review on tube 
feeding for enteral nutrition. Gastroenterology 1995; 108:1282-301.

8. Pearce CB, Duncan HD. Enteral feeding. Nasogastric, nasojejunal, percutaneous endoscopic gastrostomy, or jejunostomy: its indications and limitations. Postgrad Med J 2002;78:198-204.

9. Ciocon JO. Indications for tube feedings in elderly patients. Dysphagia 1990;5:1-5.

10. Campbell-Taylor I, Nadon GW, Sclater AL, Fisher R, Harris-Kwan J, Rosen I. Oro-esophageal tube feeding: an alternative to nasogastric or gastrostomy tubes. Dysphagia 1988;2:220-1.

11. Funahashi M, Nakajima S, Ishihara K, Nishimura F. Intermittent use of an oral catheter for feeding dysphagic children. No To Hattatsu 1985;17:3-9.

12. You DS, Chun MH, Kim HJ, Ryu JS, Song YJ, Park EJ, et al. The effectiveness of oro-esophageal tube feeding with dysphagia after brainstem stroke. J Korean Acad Rehabil Med 2011;35:27-33.

13. Kim J, Seo HG, Lee GJ, Han TR, Oh BM. The feasibility and outcome of oro-esophageal tube feeding in patients with various etiologies. Dysphagia 2015;30:6805.

14. Han TR, Paik NJ, Park JW. The follow-up of Oro-Esophageal (OE) tube feeding. J Korean Acad Rehabil Med
2001;25:58-61.

15. Bassi GS, Humphris GM, Longman LP. The etiology and management of gagging: a review of the literature. J Prosthet Dent 2004;91:459-67.

16. Davies AE, Kidd D, Stone SP, MacMahon J. Pharyngeal sensation and gag reflex in healthy subjects. Lancet 1995;345:487-8.

17. Ramsey D, Smithard D, Donaldson N, Kalra L. Is the gag reflex useful in the management of swallowing problems in acute stroke? Dysphagia 2005;20:105-7.

18. Nakajima M, Takada T, Terasaki Y, Nagano K, Naritomi $\mathrm{H}$, Minematsu K. Clinical significance of oral intake in patients with acute stroke. Dysphagia 2010;25:192-7.

19. Nakajima M, Kimura K, Inatomi Y, Terasaki Y, Nagano $\mathrm{K}$, Yonehara T, et al. Intermittent oro-esophageal tube feeding in acute stroke patients: a pilot study. Acta Neurol Scand 2006;113:36-9.

20. Halper AS, Cherney LR, Cichowski K, Zhang M. Dysphagia after head trauma: the effect of cognitivecommunicative impairments on functional outcomes. J Head Trauma Rehabil 1999;14:486-96.

21. Toscano M, Cecconi E, Capiluppi E, Vigano A, Bertora P, Campiglio L, et al. Neuroanatomical, clinical and cognitive correlates of post-stroke dysphagia. Eur Neurol 2015;74:171-7. 